\title{
Spinocerebellar ataxia type 19/22
}

INSERM

\section{Source}

INSERM. (1999). Orphanet: an online rare disease and orphan drug data base.

Spinocerebellar ataxia type 19/22. ORPHA:98772

Spinocerebellar ataxia type 19 (SCA19) is a very rare subtype of type I autosomal dominant cerebellar ataxia (ADCA type I; see this term). It is characterized by mild cerebellar ataxia, cog nitive impairment, low scores on the Wisconsin Card Sorting Test measuring executive function, myoclonus, and postural tremor. 